\title{
Predictors and Moderators of Clinical Outcomes in Adolescents with Severe Mental Disorders After an Assertive Community Treatment
}

\author{
Sébastien Urben - Vanessa Baier · Gregory Mantzouranis • Eva Pigois • \\ Caroline Graap · Fabienne Dutoit • Mélanie Cherix-Parchet · Coralie Henz • \\ Aymeric Faucherand $\cdot$ Esperanza Senent $\cdot$ Laurent Holzer
}

Published online: 21 February 2015

(C) Springer Science+Business Media New York 2015

\begin{abstract}
Previous studies have shown that stressful life events (SLEs), gender, social functioning and pretreatment severity are some of the predictors and/or moderators of treatment outcome in psychiatric care. The current study explored the effect of these predictors and moderators on the treatment outcome related to assertive community treatment (ACT) proposed to young people with severe mental disorders. 98 patients were assessed for externalizing and emotional difficulties, at admission and then at discharge of an ACT. Analyses revealed significant improvements in terms of symptomatology. In particular, regression analyses showed that pretreatment severity is a significant predictor of the outcome on emotional symptoms and is moderated by SLE on the outcome on externalizing symptoms. Furthermore, higher social functioning
\end{abstract}

S. Urben $(\bowtie) \cdot$ G. Mantzouranis

Research Unit, University Psychiatric Service for Child and

Adolescent (UPSCA), Department of Psychiatry (DP),

University Hospital (CHUV), Rue du Bugnon 25A,

1011 Lausanne, Switzerland

e-mail: Sebastien.Urben@chuv.ch

V. Baier - E. Pigois - C. Graap · F. Dutoit - A. Faucherand ·

E. Senent $\cdot$ L. Holzer

Mobile Team for Adolescent, University Psychiatric Service for Child and Adolescent (UPSCA), Department of Psychiatry (DP),

University Hospital (CHUV), Lausanne, Switzerland

M. Cherix-Parchet

Mobile Team for Child and Adolescent, Psychiatric and Psychotherapeutic Service for Child and Adolescent, Nant

Foundation, Aigle, Switzerland

C. Henz

Mobile Team for Child and Adolescent, Psychiatric Service for Child and Adolescent, Department of Psychiatry (DP),

University Hospital (CHUV), Yverdon, Switzerland proved to predict better outcome on externalizing symptoms. Our results further evidence that these factors can explain inter-individual differences in outcome related to ACT. The theoretical and clinical implications of these results are discussed.

Keywords Adolescents $\cdot$ Psychiatric disorders $\cdot$ Assertive community treatment $\cdot$ Treatment outcome $\cdot$ Stressful life events $\cdot$ Pretreatment severity $\cdot$ Moderation effect

\section{Introduction}

The developmental fragility of the adolescent period and the necessity to consider the social environment are two important specificities which have to be taken into account in order to provide appropriate care for young people in crisis and to help them recover an equilibrium [1]. However, regular models of care aren't always appropriate for these young people. Indeed, these models usually take place in clinical settings and all the provided skills and services require the patients to go a step further and apply them to "real-life" situations [2]. In addition, the need to be in a hospital environment to benefit from appropriate care makes these traditional models seem more threatening. This has a stronger impact on young people in particular, because they are generally less accustomed to hospital care.

In this context, one major advantage of assertive community treatment (ACT) models is that the interventions are provided in the adolescent's living environment (e.g. at home, in schools, in coffee shops) by health professionals wearing civilian clothes, which contributes to the creation of a greater sense of trust [3]. Moreover, the ACT teams provide services (e.g. counseling, social skills training, or 
crisis management) directly in the settings and contexts in which problems tend to arise. Other key features of the ACT are the high frequency of treatment contacts (up to twice a day, 5 days a week), the small client/staff ratio, and the extended availability for crisis intervention. Thus, ACT allows a better apprehension of the social environment of young people and the help of a case manager provides expertise in developmental aspects of this stage. Additionally, continuity of care (e.g. the involvement of the caregivers in numerous facilities), involvement of relatives, social insertion, less stigmatization and favorable cost/ benefit ratios may be considered as some of the numerous advantages of the ACT [4]. Moreover, ACT seems to be efficient at reducing the symptoms and is able to reduce the duration of inpatient care [5-11]. Thus, ACT makes it easier to maintain individuals in their community and permits better social insertion (see [1] for a review).

However, adolescents show a great interpersonal variability in their clinical outcomes following an ACT. Thus, one core question that remains unsolved is which patients are most likely to present the best outcomes after ACT. It is of the utmost importance to understand the predictors and the moderators of treatment outcome to better tailor the treatment strategy and to increase the likelihood of having the best clinical outcome. More specifically, moderators refer to factors specifying the condition under which the treatment outcome might be more or less effective [12]. Moderators modulate the treatment outcome of some patients with specific characteristics such as a factor explaining the treatment outcome only in boys or young people with past traumatism, for instance. Moderators generally precede in time both the predictors and the outcome. Whereas predictors of treatment outcome refer to factors that are not differentially linked to such outcomes depending on characteristics such as group assignment or gender [13].

In this perspective, many factors were generally reported to have an importance in predicting and moderating treatment outcomes in both traditional treatments and ACT $[14,15]$. Many studies carried out on adult samples showed that three intrapersonal factors (stressful life events, gender and pretreatment severity) and one interpersonal factor (social functioning) were particularly influential in this aspect. Studies on adolescent samples are rare, but tend to identify the same main factors as studies on adults, although not always with the same kinds of association.

Regarding stressful life events (SLEs), it was demonstrated that adolescents presenting more of these events are more prone to have psychiatric disorders [16], relationship difficulties as well as substance abuse problems [17] and disruptive behaviors [18]. The association between SLE and psychopathology seems to be moderated by gender, but mixed results were reported. Indeed, some studies have indicated that being a girl is a protective factor [19], whereas other studies found the opposite [20], in particular for internalizing symptoms [21].

Regarding the importance of SLE in treatment outcome, mixed results were observed depending on the age of the sample studied. Some studies focusing on internalizing symptoms among adults reported that more SLE are related to a better clinical outcome [22], while others didn't find any associated feature [23]. Contrary to adult populations, a study on children found that SLE were related to a poorer clinical outcome [24]. However, despite the fact that maltreated children were shown: (a) to present more externalizing symptoms like aggression [24]; (b) to present higher externalizing problems at admission in a psychiatric treatment program; (c) and to have a greater likelihood to drop out of the program [14], few other studies explored the influence of SLE on externalizing symptoms.

Additionally, mixed results were also observed regarding the importance of pretreatment severity. Studies on depressive disorder in adults treated by cognitive behavioral therapy (CBT) showed that higher pretreatment severity is predictive of better treatment outcome following CBT [25]. In contrast, other studies reported that a high number of post-traumatic stress disorder symptoms prior to treatment is related to poor outcome in veterans [26]. More closely related to our focus, a study [27] reported that the treatment outcome for children with antisocial behavior treated with CBT is predicted by lower severity of clinical dysfunction (i.e. less severe chronic symptoms of antisocial behavior, fewer symptoms across a range of psychiatric disorder).

Finally, social functioning has been reported as a significant predictor of clinical outcome in inpatient context for adolescent populations. For example, Stanley et al. [28] reported that repetitive suicide attempters showed more social and academic dysfunction. Likewise, Barter et al. [29] observed a higher rate of suicide attempts in adolescents with a history of parental loss or living outside their family home (i.e. social dimensions). More recently, King et al. [30] studied adolescents suffering from a wide range of psychiatric disorders and reported that social adjustment at baseline is a predictor of outcome in social functioning after an inpatient intake. In the context of schizophrenia research, social functioning was also observed as an important predictor of clinical outcome (i.e. negative symptoms) for adolescents [31]. Furthermore, the level of family conflict was identified as moderating treatment response in a study comparing treatment outcome of community or of clinic treatment for children with oppositional defiant disorder or conduct disorders [15].

As reported above, studies exploring the predicting and moderating effects of the factors affecting the treatment outcome in psychiatric care are very scarce among children 
and adolescents and mixed results were observed. Moreover, to the best of our knowledge, no previous study explored the role of these factors, taken together in the context of ACT on young patients. So, we would like to test whether the care provided by the ACT is equally efficient for all patients. In other words, we assessed the predicting of pretreatment severity and social functioning, and moderating effects of gender and SLE on the treatment outcome as these factors were reported to be important to understand treatment outcome in a broader context of psychiatric care. As the design of the present study consists of an open-label trial, where the treatment response (i.e. change score) contains various confounding-variables like spontaneous recovery from symptoms, for example, we have focused on understanding of clinical outcomes. These refer to important aspects for the well-being of the patients after an ACT and are less affected by confounding factors.

\section{Materials and Methods}

\section{Participants}

All patients agreeing to be followed by ACT teams were enrolled in the study. In total, 129 patients were followed by these teams. However, we only have assessment at discharge for 98 of the patients followed by the ACT teams, 31 patients having dropped out of the program, leaving $75 \%$ of the patients who were included in the analyses. Table 1 describes the sample characteristics. The mean age of the sample is 15.97 ( $\mathrm{SD}=1.58$ ).

The majority of young people followed by the ACT team were male. The proportion of each of the pathologies was similar. The majority of the young people lived with their families and no longer attended school.

Table 1 Socio-demographic data

\begin{tabular}{llc}
\hline Variable & Categories & Percentage \\
\hline Gender & Male & 57.1 \\
& Female & 42.9 \\
Disorders & Internalizing & 35.6 \\
& Externalizing & 27.4 \\
& Mix & 37.0 \\
Scolarity & No & 68.2 \\
Life's mode & Alone & 3.2 \\
& Family & 59.6 \\
& Foster family & 1.1 \\
& Educational home & 36.2 \\
\hline
\end{tabular}

Intervention

The ACT teams propose psychiatric care including several advantages such as the continuity of care, the implication of the patient's relatives and a less stigmatizing treatment. The ACT is intended for adolescents aged from 13 to 18 years suffering from severe psychiatric disorders. The multidisciplinary care team is composed of psychiatrists, social workers and nurses who all assume the role of case managers. The intervention model is an assertive, flexible, time-limited treatment, with frequent meetings taking place in the environment of the young people, with direct involvement of the family. A more detailed description of the intervention proposed by these ACT teams can be found in Baier et al. [3]. Different types of interventions (see [1]), are available: (a) Early intervention which is thought to help adolescents follow a more regular psychiatric treatment, take their medication properly or alleviate daily life difficulties (e.g. school attendance, problems with peers or stressful life events); (b) Transition case management helping the young people during a transitional period just after being discharged from hospital; (c) Care provided to barely accessible patients or those in refusal of care aiming to establish a therapeutic alliance with them; (d) Psychiatric assessment in the community (such as outpatient facilities, schools, and incarceration centers) in order to discuss the problematic situation; (e) More classical ACT through intervention in the teenagers own community assessing the needs and expectations of the family or the educative home and the young people in order to develop a therapeutic alliance.

\section{Measures}

\section{Clinical Outcome}

Symptom assessment was carried out on admission (T1) and upon discharge (T2) with the Health of the Nation Outcome Scales (Child and Adolescent Mental Health; HoNOSCA) [32]. HoNOSCA has proved to be a valid measurement tool, to be sensitive to clinical changes (e.g. [32-38]), and to have acceptable inter-rater reliability and face validity in inpatient and outpatient settings [32, 39, 40]. In this study, the assessments were carried out by trained psychiatrists, psychologists or nurses using the French version of the HoNOSCA [41]. It is composed of 15 items scored on a 5-point severity scale ranging from 'no problems' (0) to 'severe problems' (4). The measure provided by the HoNOSCA, based on the factor structure proposed by Tiffin and Rolling [42], is the mean score of its two subscales: the emotional symptoms subscale (Emo, by averaging the scores of the items $3,4,6,7,8,9,10,12,13$ ) and of the externalizing problems subscale (Ext, by 
averaging the scores of the items 1,2, 5 and 11). For all scores, higher scores represent higher difficulties. Reliability within this study, assessed with Cronbach's alphas, is of 0.709 for Emo and of 0.687 for Ext, which is acceptable.

\section{Pretreatment Severity}

Pretreatment severity was assessed at admission with the Clinical Global Impression-Severity of illness scale (CGI) [43]. CGI is a scale that is commonly used as a primary outcome measure in many psychiatric studies [44-46]. This scale has numerous advantages, including established utility in psychiatric research, sensitivity to change, quick and simple administration and reliable measure [47]. The caregivers filled the CGI in with a unique score ranging from 1 (normal) to 7 (amongst the most severely ill patients), according to the caregiver's experience of patients suffering from pathologies.

\section{Social Functioning}

Social functioning was assessed at admission with the Social and Occupational Functioning Assessment Scale (SOFAS) [48]. The SOFAS is a reliable and valid assessment of the Axe V of the DSM classification [49]. This scale was rated by the caregivers according a unique score of between 0 and 100 in function of pre-established criteria. For instance a score of "1" refers to "persistent hygiene problems" and a score of "100" to "superior functioning in a wide range of activities".

\section{Stressful Life Events (SLE)}

The SLE taken into account in this study were based on the main SLE studied in the literature [50] as well as on the ICD-10 criteria [51]. They were assessed by the caregivers and were related to the following events: adoption, parting of the parents, parental dispute, sexual abuse, physical abuse, death of relatives, mental disorders of parents, mental disorders of siblings, and migration. The caregivers specified for each SLE whether it occurred previously to the patient's admission for treatment or not. Because previous studies (e.g. [52]) observed a cumulative effect of SLE on the treatment outcome, the score used in our subsequent analyses refers to the sum of the SLE.

\section{Ethical Considerations}

This study is part of a larger project referring to an observational study that examines the benefits of implementing ACT teams for different age spans (i.e. adults and adolescents) in the state of Vaud in Switzerland. The global project aims to assess the quality of ACT care. This type of care is designed for patients who, for most part, refuse regular psychiatric care. Additionally, ACT teams don't refer to exclusive treatment options. Thus, the possibility to be followed by ACT teams do not prevent other treatment options.

Within this context, when proposed to be followed by ACT teams, all young people and their guardians were informed that routine clinical assessments were going to be made and eventually published. Thus, they had the possibility to refuse or give consent for their data to be used for scientific purposes. A refusal did not have any influence on the proposed treatment. The procedure was approved by the local Ethic Committee.

\section{Data Analyses}

The distribution of the outcome variables (HoNOSCA scores) revealed that the data distribution conformed to a "normal" (or Gaussian) distribution and did not comprise outliers. Therefore, the clinical outcome was assessed with paired sample tests of Student by comparing admission scores to discharge scores.

Then, we assessed the predicting and moderating effects. Specifically, the predictors refer to the direct effect of a variable on the outcome. In contrast, moderators (always preceding in time the predicting variable and the outcome) modulate the clinical outcome. According to this definition of moderation, we hypothesized that SLE or gender might moderate the clinical outcome. Therefore, we assessed the effect of the predictors (direct effect of social functioning, pretreatment severity, gender and SLE) and the interaction effects between these factors and SLE and gender (coded as 0 for female and 1 for male), which refer to moderation effects on the clinical outcome. Above all, we first computed correlational analyses and then multiple regression analyses on the clinical outcome scores (HoNOSCA scores at discharge). To compute the moderation effect, we first centered the variables by subtracting the sample's mean from each individual score. We then multiplied each one of our postulated moderators with the other predictors, before adding them to the regression model.

To sum up, we computed multiple regression analyses with both predicting factors (simple effects: SOFAS, CGI, gender and SLE) and moderation effects of gender and SLE (interaction effects: Gender $\times$ SLE, Gender $\times$ SOFAS, Gender $\times$ CGI, SLE $\times$ SOFAS, SLE $\times$ CGI) . When moderation effects were significant, we plotted the interaction graph in order to illustrate them, following Preacher et al.'s [53] guidelines. For all regression analyses, the variance inflation factor (VIF) and the tolerance were inspected revealing no problem of collinearity. In addition, the residuals distributions of the regression analyses followed a Gaussian (or "normal") distribution and no outliers were observed. 


\section{Results}

The mean length of the ACT was of 189.72 days $(\mathrm{SD}=131.26)$. The SLE were represented as follows: parting of the parents $(60.2 \%)$, parental dispute $(41.8 \%)$, death of relatives $(37.8 \%)$, physical abuse $(33.7 \%)$, mental disorders of parents $(34.7 \%)$, migration (16.3\%), sexual abuse (13.3\%), adoption (8.2\%), and mental disorders of siblings $(6.1 \%)$. The sum of SLE is on average of 2.84 and SD is of 2.05 , with scores ranging from 0 to 8 . The SOFAS score (social functioning) is on average of 46.46 and SD of 14.44 whereas the CGI score (pretreatment severity) is on average of 4.23 and SD of 1.20 . Table 2 sums up the scores of the clinical outcomes. Paired sample $t$ tests revealed that the Emo score $(t(96)=5.66$, $p<0.001, d=0.57)$ and the Ext score $(t(97)=2.48$, $p<0.05, d=0.25$ ) of the HoNOSCA were significantly improved at $\mathrm{T} 2$.

Table 3 presents the correlations between the clinical outcome and the predictors. The results of the multiple regression models trying to explain variance in clinical outcomes are summed up in Table 4. The model significantly explained the HoNOSCA Emo score at discharge $\left(F(9,81)=3.06, p<0.01, R^{2}=0.25\right)$. In particular, lower pretreatment severity (lower CGI score at admission) was related to lower HoNOSCA Emo score at discharge (lower emotional difficulties). The model also explained a significant part of the variance of the HoNOSCA Ext score $\left(F(9,81)=3.98, p<0.001, R^{2}=0.31\right)$. In particular, a higher SOFAS score was related to a lower HoNOSCA Ext score at discharge (lower externalizing problems).

Table 2 Descriptives of the HoNOSCA scores

\begin{tabular}{lrrrrrr}
\hline & \multicolumn{2}{c}{ Admission } & & \multicolumn{2}{c}{ Discharge } \\
\cline { 2 - 3 } & Mean & SD & & Mean & SD \\
\hline HoNOSCA Ext score & 6.11 & 3.12 & & 5.40 & 3.28 \\
HoNOSCA Emo score & 14.87 & 3.96 & & 12.24 & 5.47 \\
\hline
\end{tabular}

Additionally, the interaction between SLE and CGI (pretreatment severity) explained the HoNOSCA Ext score at discharge. Figure 1 illustrates this moderation effect.

The moderation could be understood as follows: when young people have not been confronted with many SLE previously in life, the pretreatment severity has less influence on the HoNOSCA Ext score (externalizing symptoms) at discharge. In contrast, when they have been confronted with many SLE during their life the pretreatment severity takes on a great importance. Indeed, if both SLE and pretreatment scores are high (worse situation) the HoNOSCA Ext score is higher (worse clinical outcome) at discharge. In other words, SLE have a moderation effect on the influence of pretreatment severity on the outcome of externalizing symptoms.

\section{Discussion}

This study aims to understand the prediction and moderation effects of SLE, gender, pretreatment severity and social functioning on the clinical outcome following an ACT. First, we observed a significant improvement related to ACT. Additionally, we observed that young people presenting the highest pretreatment severity showed the poorest treatment outcome regarding the emotional symptoms. Furthermore, results revealed that the poorest treatment outcome for externalizing problems is predicted by poor psychosocial functioning and pretreatment severity whose influence is moderated by SLE.

First, results revealed a reduction of both emotional and externalizing symptoms after ACT. These results showing a global alleviation of young people's difficulties are in line with previous studies showing the efficacy of the ACT for adults (e.g. [54]) or for young people (e.g. [3, 55]). It is worth mentioning that the specificity of ACT is to take into account the social environment to provide the most appropriate care to young people in crisis in order to help them recover an optimal balance [1]. This allows them to

Table 3 Correlation analyses

\begin{tabular}{|c|c|c|c|c|c|c|}
\hline Variables & 1. HoNOSCA Emo score & 2. HoNOSCA Ext score & 3. Gender & 4. SLE & 5. CGI & 6. SOFAS \\
\hline 1. & - & $0.722 * *$ & -0.075 & 0.137 & $0.441 * *$ & $-0.388 * *$ \\
\hline 2. & & - & 0.023 & 0.123 & $0.404 * *$ & $-0.419 * *$ \\
\hline 3. & & & - & $-0.321 * *$ & 0.026 & -0.004 \\
\hline 4. & & & & - & 0.037 & -0.029 \\
\hline 5. & & & & & - & $-0.532 * *$ \\
\hline 6. & & & & & & - \\
\hline
\end{tabular}

Bravais-Pearson coefficients of correlations. HoNOSCA Emo score at discharge; HoNOSCA Ext score at discharge; Gender: female 0 and male 1; SLE: Stressful life events; SOFAS score at admission

$* * p<0.01$ 
Table 4 Sums up of the multiple regression analyses
Bold values indicate the significant predictors

HoNOSCA Emo score at discharge; HoNOSCA Ext score at discharge; SLE: Stressful life events; SOFAS score at admission

\begin{tabular}{|c|c|c|c|c|c|c|}
\hline Dependent variable & Predictor variables & $B$ & $S E B$ & $\beta$ & $t$ & $p$ \\
\hline \multirow[t]{10}{*}{ HoNOSCA Emo score } & (Constant) & 5.12 & 5.45 & & 0.94 & 0.350 \\
\hline & CGI & 1.86 & 0.70 & 0.44 & 2.64 & 0.010 \\
\hline & SOFAS & -0.02 & 0.06 & -0.05 & -0.27 & 0.788 \\
\hline & Gender & -0.49 & 1.06 & -0.05 & -0.46 & 0.649 \\
\hline & SLE & 0.09 & 0.40 & 0.03 & 0.22 & 0.826 \\
\hline & Gender $\times$ SLE & -0.01 & 0.53 & 0.00 & -0.03 & 0.978 \\
\hline & Gender $\times$ CGI & -0.93 & 1.03 & -0.17 & -0.90 & 0.370 \\
\hline & Gender $\times$ SOFAS & -0.12 & 0.09 & -0.25 & -1.24 & 0.220 \\
\hline & $\mathrm{SLE} \times \mathrm{CGI}$ & 0.31 & 0.32 & 0.14 & 0.95 & 0.344 \\
\hline & SLE $\times$ SOFAS & 0.00 & 0.03 & 0.00 & -0.01 & 0.990 \\
\hline \multirow[t]{10}{*}{ HoNOSCA Ext score } & (Constant) & 8.79 & 3.30 & & 2.67 & 0.009 \\
\hline & CGI & 0.30 & 0.43 & 0.12 & 0.71 & 0.477 \\
\hline & SOFAS & -0.11 & 0.04 & -0.49 & -2.85 & 0.006 \\
\hline & Gender & 0.32 & 0.64 & 0.05 & 0.49 & 0.623 \\
\hline & SLE & 0.09 & 0.24 & 0.05 & 0.37 & 0.715 \\
\hline & Gender $\times$ SLE & -0.06 & 0.32 & -0.03 & -0.20 & 0.846 \\
\hline & Gender $\times$ CGI & 0.87 & 0.62 & 0.25 & 1.39 & 0.167 \\
\hline & Gender $\times$ SOFAS & 0.09 & 0.06 & 0.29 & 1.51 & 0.135 \\
\hline & SLE $\times$ CGI & 0.47 & 0.19 & 0.35 & 2.42 & 0.018 \\
\hline & SLE $\times$ SOFAS & 0.01 & 0.02 & 0.12 & 0.85 & 0.399 \\
\hline
\end{tabular}

have the best possible trajectories in their specific developmental period [4] which certainly helps to alleviate their symptomatology.

Second, we observed that a better outcome regarding emotional difficulties is related to a lower pretreatment severity which is in line with previous results [26, 27]. In the same vein, we observed that a better pretreatment social functioning is related to a higher treatment outcome. One

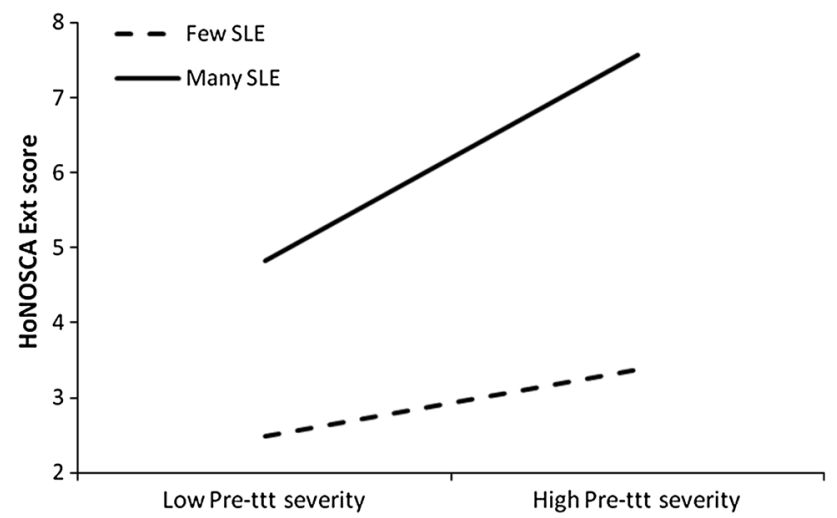

Fig. 1 Moderation effect of stressful life events (SLE) on the importance of pretreatment severity in explaining the outcome on externalizing problems. Note Low Pre-ttt severity: -1 standard deviation (SD) of the HoNSOCA Ext score at admission, High Pre-ttt severity: +1 SD of the HoNSOCA Ext score at admission. Not many SLE: -1 SD of the mean of the sum of Stressful life events (SLE); Many SLE: +1 SD of the mean of the sum of SLE. Externalizing problems at discharge: HoNOSCA Ext score at discharge interesting hypothesis that could help us understand these results is the stress generation hypothesis [56], which postulates that individuals with psychiatric disorders encounter high rates of acute and chronic stress which are dependent, at least in part, on their own actions. Thus, adopting this perspective, individuals with a higher pretreatment severity at admission in the ACT may be exposed to higher levels of stress from the beginning and also during the whole psychotherapeutic treatment which reduces the quality of interactions with the caregivers. Moreover, as the therapeutic alliance is one of the most important factors explaining the outcome (e.g. [57]), we could imagine that a reduction of therapeutic alliance would impair an optimal alleviation of symptoms.

Furthermore, we observed an interaction effect between pretreatment severity and SLE on the outcome regarding externalizing problems. In particular, we observed that when young people were confronted to many SLE, pretreatment severity had a greater influence on outcome. However, if young people were not confronted to many SLE, pretreatment severity did not have an influence on clinical outcome. Thus, SLE have a moderation effect on the relation between pretreatment severity and outcome about externalizing problems. In previous literature, SLE were related to the presence of many psychiatric disorders [16]. So, it was proposed that being confronted to many SLE early in life might deeply impair the maturation of the stress response system, the development of emotion regulation and the formation of basic relationship schemas 
(e.g. [58]). Thus, being exposed to SLE in childhood may have long-term negative consequences on development such as social malfunctioning (e.g. externalizing symptoms). As exposed above, stress generation phenomena may impair the therapeutic alliance formation among young people, which results in a worse clinical outcome. Thus, we may hypothesize that such phenomenon moderates the influence of pretreatment severity on treatment outcome about externalizing problems.

Finally, social functioning was observed to be a predictor of externalizing symptoms. In particular, we reported that a higher social functioning at admission of ACT was related to a better outcome regarding externalizing symptoms. Likewise, previous studies demonstrated that higher social functioning is related to fewer suicide attempts in a context of inpatients treatment $[28,29]$ or that it moderates the outcome of externalizing symptoms, when comparing ACT to a clinical treatment [15]. Regarding the focus of ACT on young people's psychosocial environment and the use of resources in this context, we may hypothesize that young people with a better social functioning are more able to use the resources of their environment and to interact in a better way, which helps them alleviate their externalizing problems.

Regarding the clinical implications, first, adopting the stress generation theory seems to help us better understand or apprehend our results. Thus, if such processes are in action during ACT, one might suppose that relaxation sessions or a focus on stress reduction might help the young people to benefit more from it. Second, regarding the importance of social functioning in the alleviation of externalizing symptoms, social skill training for young people with low social functioning at admission in ACT might help them to alleviate their externalizing difficulties more efficiently. Third, our results suggest that SLE moderate the clinical outcome. Thus, it is important to assess these aspects and furthermore to propose specialized treatment strategies adapted for these patients. For instance, as this moderation is linked to the pretreatment severity, it is essential that both aspects (multiple SLE and high pretreatment severity) are presented at admission to consider specific treatment strategies, focusing for instance on the reduction of the symptoms and the understanding of the impact of SLE.

This study refers to a retrospective study with all the potential limitations associated with such a design. For instance, the assessment of clinical outcomes has been made only from the caregivers' point of view. The study could benefit from adopting a multiple informant perspective, including the patient's for example. Moreover, further studies might benefit from using standardized questionnaires or interviews to collect information on SLE. The absence of a randomized control group did not allow to formally prove the efficacy of the ACT. Indeed, with our design it is not possible to control for the spontaneous improvement due to the passing of time.

\section{Summary}

Adolescents show a great interpersonal variability in their clinical outcomes following an ACT. Thus, one core question that remains unsolved is which patients are most likely to present the best outcomes after ACT. Previous research in psychiatric care demonstrated the role of stressful life events, gender, pretreatment severity and social functioning in predicting and moderating the treatment outcome. Thus, the current study assesses the role of these dimensions on the treatment outcome of an ACT for adolescents with severe psychiatric disorders. Results determine that pretreatment severity predicts the outcome on emotional symptoms whereas stressful life events moderate the influence of pretreatment severity on the outcome on externalizing symptoms. Furthermore, outcome on externalizing symptoms is also predicted by social functioning. These results can help to define finer therapeutic strategies. Indeed, reducing the severity of the symptoms before entering an ACT program might have a beneficial effect on the internalizing symptoms. Likewise, enhancing social functioning by social skill training, for example, might help to improve the externalizing symptoms. Thus, the present study has shed some light on several important factors influencing the treatment outcome related to ACT.

\section{Conflict of interest None.}

\section{References}

1. Graap C, Urben S, Baier V, Senent E, Holzer L, Pigois E (2014) Equipe mobile pour Adolescents. In: Holzer L (ed) Psychiatrie de l'enfant et de l'adolescent, une approche basée sur les preuves. De Boek, Belgium

2. Phillips SD, Burns BJ, Edgar ER, Mueser KT, Linkins KW, Rosenheck RA et al (2001) Moving assertive community treatment into standard practice. Psychiatr Serv 52:771-779

3. Baier V, Favrod J, Ferrari P, Koch N, Holzer L (2013) Early tailored assertive community case management for hard-to-engage adolescents suffering from psychiatric disorders: an exploratory pilot study. Early Interv Psychiatry 7:94-99

4. Urben S, Baier V, Geiser E, Senent E, Dutoit F, Holzer L (2013) [Mobile team for Adolescents: a pilot study assessing efficacy and impact of adverse life events] FRENCH. Swiss Arch Neurol Psychiatr 164:206-2011

5. Henggeler SW, Clingempeel WG, Brondino MJ, Pickrel SG (2002) Four-year follow-up of multisystemic therapy with substance-abusing and substance-dependent juvenile offenders. J Am Acad Child Adolesc Psychiatry 41:868-874

6. Henggeler SW, Pickrel SG, Brondino MJ (1999) Multisystemic treatment of substance-abusing and dependent delinquents: outcomes, treatment fidelity, and transportability. Ment Health Serv Res 1:171-184 
7. Henggeler SW, Rowland MD, Halliday C, Sheidow AJ, Ward DM, Randall J et al (2003) One year follow-up of multisystemic therapy as an alternative to the hospitalisation of youths in psychiatric crisis. J Am Acad Child Adolesc Psychiatry 42:543-551

8. Mattejat F, Hirt BR, Wilken J, Schmidt MH, Remschmidt H (2001) Efficacy of inpatient and home treatment in psychiatrically disturbed children and adolescents. Follow-up assessment of the results of a controlled treatment study. Eur Child Adolesc Psychiatry 10(Suppl 1):I71-I79

9. Rowland MD, Halliday-Boykins C, Henggeler SW, Cunningham PB, Lee T, Kruesi M et al (2005) A randomised trial of multisystemic therapy with Hawaii's Felix class youth. J Emot Behav Disorders 13:13-23

10. Sheidow AJ, Bradford WD, Henggeler SW, Rowland MD, Halliday-Boykins C, Schoenwald SK et al (2004) Treatment costs for youths receiving multisystemic therapy or hospitalization after a psychiatric crisis. Psychiatr Serv 55:548-554

11. Huey SJ Jr, Henggeler SW, Rowland MD, Halliday-Boykins CA, Cunningham PB, Pickrel SG et al (2004) Multisystemic therapy effects on attempted suicide by youths presenting psychiatric emergencies. J Am Acad Child Adolesc Psychiatry 43:183-190

12. Kraemer HC, Wilson GT, Fairburn CG, Agras WS (2002) Mediators and moderators of treatment effects in randomized clinical trials. Arch Gen Psychiatry 59:877-883

13. Shelleby EC, Shaw DS (2014) Outcomes of parenting interventions for child conduct problems: a review of differential effectiveness. Child Psychiatry Hum Dev 45:628-645

14. Lau AS, Weisz JR (2003) Reported maltreatment among clinicreferred children: implications for presenting problems, treatment attrition, and long-term outcomes. J Am Acad Child Adolesc Psychiatry 42:1327-1334

15. Shelleby EC, Kolko D (2013) Predictors, moderators, and treatment parameters of community and clinic-based treatment for child disruptive behavior disorders. J Child Fam Stud. doi:10. 1007/s10826-013-9884-1

16. Tiet QQ, Bird HR, Hoven CW, Moore R, Wu P, Wicks J et al (2001) Relationship between specific adverse life events and psychiatric disorders. J Abnorm Child Psychol 29:153-164

17. Somasundaram DJ, Sivayokan S (1994) War trauma in a civilian population. Br J Psychiatry 165:524-527

18. Finkelhor D (1995) The vitimization of children: a developmental perspective. Am Orthopsychiatr Assoc 65:177-193

19. Luthar SS, Doernberger CH, Zigler E (1993) Resilience is not a unidimensional construct: insights from a prospective study of inner-city adolescents. Dev Psychopathol 5:703-717

20. Werner EE, Smith RS (1992) Overcoming the odds: high risk children from birth to adulthood. Cornell University Press, Ithaca

21. Leadbeater BJ, Kuperminc GP, Blatt SJ, Hertzog C (1999) A multivariate model of gender differences in adolescents' internalizing and externalizing problems. Dev Psychol 35:1268-1282

22. Reno RM, Halaris AE (1990) The relationship between life stress and depression in an endogenous sample. Compr Psychiatry $31: 25-33$

23. Bock C, Bukh JD, Vinberg M, Gether U, Kessing LV (2009) Do stressful life events predict medical treatment outcome in first episode of depression? Soc Psychiatry Psychiatr Epidemiol 44:752-760

24. Marchand WR, Wirth L, Simon C (2005) Adverse life events and pediatric bipolar disorder in a community mental health setting. Community Ment Health J 41:67-75

25. Button KS, Wiles NJ, Lewis G, Peters TJ, Kessler D (2012) Factors associated with differential response to online cognitive behavioural therapy. Soc Psychiatry Psychiatr Epidemiol 47:827-833

26. Perconte ST, Griger ML (1991) Comparison of successful, unsuccessful, and relapsed Vietnam veterans treated for posttraumatic stress disorder. J Nerv Ment Dis 179:558-562
27. Kazdin AE, Crowley MJ (1997) Moderators of treatment outcome in cognitively based treatment of antisocial children. Cogn Ther Res 21:185-207

28. Stanley EJ, Barter JT (1970) Adolescent suicidal behavior. Am J Orthopsychiatry 40:87-96

29. Barter JT, Swaback DO, Todd D (1968) Adolescent suicide attempts. A follow-up study of hospitalized patients. Arch Gen Psychiatry 19:523-527

30. King CA, Hovey JD, Brand E, Ghaziuddin N (1997) Prediction of positive outcomes for adolescent psychiatric inpatients. J Am Acad Child Adolesc Psychiatry 36:1434-1442

31. Meng H, Schimmelmann BG, Mohler B, Lambert M, Branik E, Koch E et al (2006) Pretreatment social functioning predicts 1-year outcome in early onset psychosis. Acta Psychiatr Scand 114:249-256

32. Brann P, Coleman G, Luk E (2001) Routine outcome measurement in a child and adolescent mental health service: an evaluation of HoNOSCA. The Health of the Nation Outcome Scales for Children and Adolescents. Aust N Z J Psychiatry 35:370-376

33. Gowers SG, Harrington RC, Whitton A, Beevor A, Lelliott P, Jezzard R et al (1999) Health of the Nation Outcome Scales for Children and Adolescents (HoNOSCA). Glossary for HoNOSCA score sheet. Br J Psychiatry 174:428-431

34. Gowers SG, Harrington RC, Whitton A, Lelliott P, Beevor A, Wing J et al (1999) Brief scale for measuring the outcomes of emotional and behavioural disorders in children. Health of the Nation Outcome Scales for children and Adolescents (HoNOSCA). Br J Psychiatry 174:413-416

35. Harnett PH, Loxton NJ, Sadler T, Hides L, Baldwin A (2005) The Health of the Nation Outcome Scales for Children and Adolescents in an adolescent in-patient sample. Aust N Z J Psychiatry 39:129-135

36. Iachina M, Bilenberg N (2012) Measuring reliable change of emotional and behavioural problems in children. Psychiatry Res 200:867-871

37. Kisely S, Campbell LA, Cartwright J, Cox M, Campbell J (2010) Do the Health of the Nation Outcome Scales measure outcome? Can J Psychiatry 55:431-439

38. Brann P, Coleman G (2010) On the meaning of change in a clinician's routine measure of outcome: HoNOSCA. Aust N Z J Psychiatry 44:1097-1104

39. Gowers S, Levine W, Bailey-Rogers S, Shore A, Burhouse E (2002) Use of a routine, self-report outcome measure (HoNOSCA-SR) in two adolescent mental health services. Health of the Nation Outcome Scale for Children and Adolescents. Br J Psychiatry 180:266-269

40. Pirkis JE, Burgess PM, Kirk PK, Dodson S, Coombs TJ, Williamson MK (2005) A review of the psychometric properties of the Health of the Nation Outcome Scales (HoNOS) family of measures. Health Qual Life Outcomes 3:76

41. Holzer L, Tchemadjeu IK, Plancherel B, Bolognini M, Rossier V, Chinet L et al (2006) Adolescent Drug Abuse Diagnosis (ADAD) vs. Health of Nation Outcome Scale for Children and Adolescents (HoNOSCA) in clinical outcome measurement. J Eval Clin Pract 12:482-490

42. Tiffin PA, Rolling K (2012) Structure of the Health of the Nation Outcome Scales for Children and Adolescents: an ordinal factor analysis of clinician ratings of a sample of young people referred to community mental health services. Psychiatry Res 197:154-162

43. Guy W (1976) ECDEU Assessment manual. US Department of Health, Education and Welfare, Rockville

44. Leon AC, Shear MK, Klerman GL, Portera L, Rosenbaum JF, Goldenberg I (1993) A comparison of symptom determinants of patient and clinician global ratings in patients with panic disorder and depression. J Clin Psychopharmacol 13:327-331 
45. Spearing MK, Post RM, Leverich GS, Brandt D, Nolen W (1997) Modification of the clinical global impressions (CGI) Scale for use in bipolar illness (BP): the CGI-BP. Psychiatry Res 73:159-171

46. Zaider TI, Heimberg RG, Fresco DM, Schneier FR, Liebowitz MR (2003) Evaluation of the clinical global impression scale among individuals with social anxiety disorder. Psychol Med 33:611-622

47. Berk M, Ng F, Dodd S, Callaly T, Campbell S, Bernardo M et al (2008) The validity of the CGI severity and improvement scales as measures of clinical effectiveness suitable for routine clinical use. J Eval Clin Pract 14:979-983

48. Goldman HH, Skodol AE, Lave TR (1992) Revising axis V for DSM-IV: a review of measures of social functioning. Am J Psychiatry 149:1148-1156

49. American Psychiatric Association (2013) Diagnostic and statistical manual of mental disorders, 5th edn. American Psychiatric Publishing, Arlington

50. Pitzner JK, Drummond PD (1997) The reliability and validity of empirically scaled measures of psychological/verbal control and physical/sexual abuse: relationship between current negative mood and a history of abuse independent of other negative life events. J Psychosom Res 43:125-142

51. World Health Organisation (1992) International statistical classification of diseases and related health problems, 10th revision (ICD-10). WHO, Geneva
52. Jensen PS, Richters J, Ussery T, Bloedau L, Davis H (1991) Child psychopathology and environmental influences: discrete life events versus ongoing adversity. J Am Acad Child Adolesc Psychiatry 30:303-309

53. Preacher KJ, Curran PJ, Bauer DJ (2006) Computational tools for probing interaction effects in multiple linear regression, multilevel modeling, and latent curve analysis. J Educ Behav Stat 31:437-448

54. Rosen A, Mueser KT, Teesson M (2007) Assertive community treatment-issues from scientific and clinical literature with implications for practice. J Rehabil Res Dev 44:813-825

55. Schley C, Yuen K, Fletcher K, Radovini A (2012) Does engagement with an intensive outreach service predict better treatment outcomes in 'high-risk' youth? Early Interv Psychiatry 6:176-184

56. Conway CC, Hammen C, Brennan PA (2012) Expanding stress generation theory: test of a transdiagnostic model. J Abnorm Psychol 121:754-766

57. Eltz MJ, Shirk SR, Sarlin N (1995) Alliance formation and treatment outcome among maltreated adolescents. Child Abuse Negl 19:419-431

58. Watamura SE, Donzella B, Kertes DA, Gunnar MR (2004) Developmental changes in baseline cortisol activity in early childhood: relations with napping and effortful control. Dev Psychobiol 45:125-133 